This document is published in:

IEEE Aerospace and Electronic Systems Magazine (2013). 28(2), 4-17.

DOI: http://dx.doi.org/10.1109/MAES.2013.6477864

(C) 2013 IEEE. Personal use of this material is permitted. Permission from IEEE must be obtained for all other uses, in any current or future media, including reprinting/republishing this material for advertising or promotional purposes, creating new collective works, for resale or redistribution to servers or lists, or reuse of any copyrighted component of this work in other works. 


\title{
ATC Trajectory Reconstruction for Automated Evaluation of Sensor and Tracker Performance
}

\author{
Juan Besada ${ }^{1 *}$, Andrés Soto ${ }^{1}$, Gonzalo de Miguel $^{1}$, Jesús García ${ }^{2 \dagger}$, and Emmanuel Voet ${ }^{3 \neq}$ \\ ${ }^{1}$ Polytechnic University of Madrid. GPDS-CEDITEC, C-321. ETSI Telecomunicación, Avenida Complutense 30, Madrid, 28040, Spain \\ ${ }^{2}$ Universidad Carlos III de Madrid, Avd. De la Universidad Carlos III, 22, 28270 Madrid, Spain \\ ${ }^{3}$ EUROCONTROL. DNM/COO/DCO/SRS, 96 Rue de la Fusee, 1130 Brussels, Belgium \\ e-mail: *besada@grpss. ssr.upm.es † jgherrer@inf.uc3m.es ${ }^{*}$ emmanuel.voet@erocontrol.int
}

\section{INTRODUCTION}

Currently most air traffic controller decisions are based on the information provided by the ground support tools provided by automation systems, based on a network of surveillance sensors and the associated tracker. To guarantee surveillance integrity, it is clear that performance assessments of the different elements of the surveillance system are necessary. Due to the evolution suffered by the surveillance processing chain in the recent past, its complexity has been increased by the integration of new sensor types (e.g., automatic dependent surveillance-broadcast [ADS-B] [1], Mode $S$ radars [2], and wide area multilateration [WAM] [3]), data link applications, and networking technologies. With new sensors, there is a need for system-level performance evaluations as well as methods for establishing assessment at each component of the tracking evaluation.

Initially, the focus of surveillance assessment was placed on the performance evaluation of sensors (mainly radars). There are systems that predict the performances of sensors based on their modeling. Some current examples of systems of this type are the computer-aided radar performance evaluation tool (CARPET) for assessment and design of surfacebased radars or the Radar Support System (RSS) for siting radars and predicting their performance [4].

Other systems aim at evaluating the actual operational behavior of a sensor, which in some cases implies the use of dedicated hardware for their evaluations. In this group we can find radar analysis support system for sites (RASS-S), a radar manufacturer-independent system for evaluating the different elements of radar, or a radar monitoring display
(RMD), which also has the capability of processing any surveillance data. In general, those approaches are extensions of radar data acquisition converters (RADAC) such as the one described in [5], which is a radar data acquisition and analysis platform.

The complete assessment of multisensor surveillance system performance is a complex problem. In fact, there is not a single figure of merit describing the quality of such a system as described in [6] or [7]. So assessing it would need defining metrics over different aspects as

- sensor detection (false alarms, splits, losses of detections, measurement rates, sensor coverage, etc.);

- sensor measurement error (biases, noise variances, etc.);

- monosensor and multisensor tracks initiation (initiation delays, multisensor system coverage, etc.);

- association (incorrect associations);

- tracking accuracy (track RMS errors in horizontal and vertical directions, maneuvering biases);

- tracking robustness (appearance of gaps, track splitting, near track mixing, etc.);

- mode of flight (MoF) detection (uniform segments, turns, speed changes, etc.);

- and timeliness in the kinematic status information (maneuvering detection delay and track deletion and coasting statistics). 
The realistic evaluation of these performance metrics is a complex problem. Test flights may be used for evaluation purposes, but they are quite expensive, so they are normally avoided. However, test flights can be used to confirm that an existing problem has been solved.

Another option is simulation, which has the advantage of reproducing most typical situations but normally lacks unexpected and random effects that normally appear while processing real data. This technique is mainly used for tracker software design and testing.

Much effort has been devoted in recent years to the analysis and performance prediction of different aspects of

multisensor-multitarget tracking and data fusion systems, as can be seen in [8]-[12]. Those efforts allow the prediction on nominal situations of the expected quality of the data fusion system. Nevertheless, due to data fusion complex and nonlinear behavior and to the necessary simplifications for the analysis, they are not suitable to assess data fusion quality with the same accuracy as real traffic-based procedures.

An important problem of both the simulation and analytic approaches is the dependence of the data fusion metrics on the actual evaluation scenario, as acknowledged in [13]. A real data-based assessment, if the available traffic is representative of typical operations, is less prone to the definition of nonrealistic scenarios for the evaluation. At the same time, it lacks the potential of the evaluation in unusual but relevant (especially from the safety point of view) situations.

Therefore, exploiting the real traffic in a given environment (opportunity traffic) is the key for operational ATC system performance assessment as it is affordable and can be used to test the system in realistic operational conditions. Among real traffic-based systems, there is a further classification between the systems that work online, which are mainly focused on sensor performance analysis to ensure surveillance integrity, and the offline ones used to assess both sensor and surveillance system performances. The difference is mainly based on the usable processing algorithms, which in the second case are batch-processing systems capable of taking advantage of future measurements. An example of the first class is one of the modes of operation of the analysis working position (AWP) product [14], which aims at covering the whole functionality of an online tracking quality control infrastructure.

Considering the offline systems devoted to analyze the performance of the multisensor surveillance system, we can highlight RAPS-3, AWP [14], or the system to be described in this paper (i.e., SASS-C). Thus, EUROCONTROL SASS-C is the most complete toolbox for assessment of operational surveillance systems.

The approach followed by SASS-C is based on the creation of reference trajectories by smoothing the measurements available in recorded data sets. Those reference trajectories can be used as "ground truth" and become the basis for automatic evaluation of both sensors and multiradarmultisensor real-time data processing. The performance evaluation procedure is described in Figure 1.

Previous versions of SASS-C were centered on radar sensor and tracker evaluation [15], [16], with reconstruction procedures and figures of merit tailored to this problem. As mentioned, new sensors are being deployed for ATC surveillance. Therefore, EUROCONTROL decided to upgrade their system and include new sensors and new types of evaluation, following a modular and scalable architecture.

SASS-C and opportunity trajectory reconstruction (OTR) are employed in this discussion as a complete example of the application of advanced smoothing and trajectory reconstruction techniques for multisensor-multitarget tracker assessment. Variations of this approach could be used for many other multisensor-multitarget applications, such as defense radar networks, coastal or airport surveillance, and fleet management systems. Parts of the complete architecture and performance evaluation philosophy can also be translated to other data fusion and tracking applications, such as navigation or localization.

Finally, using ideas like those in [17] or [18], a system similar to SASS-C might be used to assess the quality and integrity of the lower levels of data fusion with a short delay. Then, it could improve the quality of the online data fusion system deriving tracks by adequately characterizing aspects

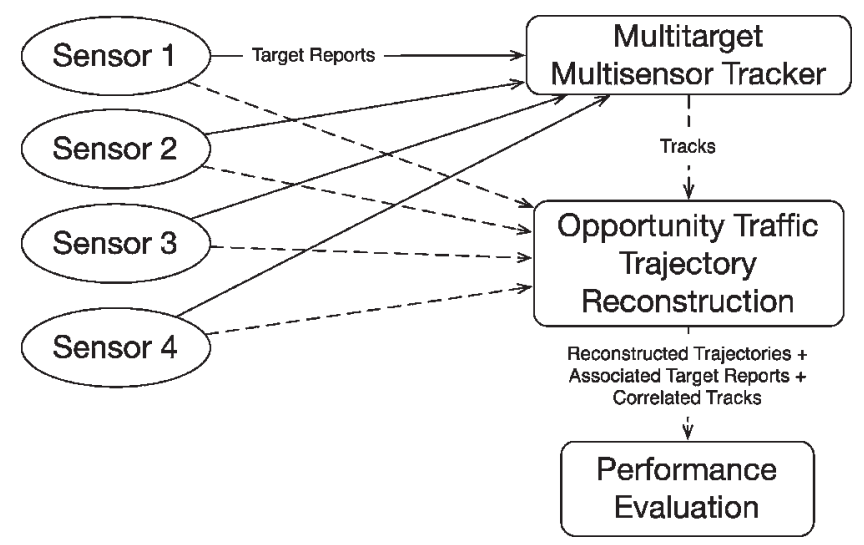

Figure 1.

Sensor and tracking assessment based on opportunity traffic. 


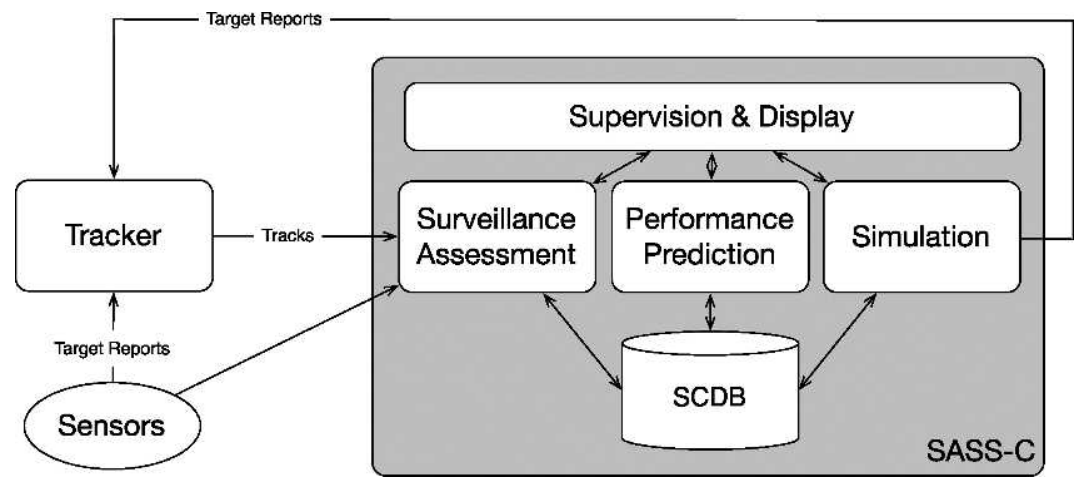

Figure 2.

SASS-C functions.

such as sensor biases or noises, false alarm levels, etc. In the current ATC surveillance architecture, this kind of adaptation process is manual.

\section{SASS-C DESCRIPTION}

SASS-C as a whole implements three main functions, as can be seen in Figure 2:

- Surveillance infrastructure performance assessment or surveillance assessment in Figure 3, to be described further in this paper, and introduced above.

- Performance prediction: it calculates the physical coverage, the probability of detection, the positioning accuracy, and an operational coverage using theoretical models.

- Simulation: it generates a realistic but synthetic surveillance data set to be fed into real-time online trackers for their assessment in a controlled environment.

The three main functions share the use of a common database (SASS-C database [SCDB]) and are controlled through a common supervision and display module (SDM) compris- ing a generic display for raw data, statistical computations and investigation results, generic reporting mechanism, and generic and highly extensible supervision.

The new modular design for surveillance assessment was built by splitting overall SASS-C v7 assessment functionality in a set of three cooperating modules that can be invoked through actions in the supervision module, as depicted in Figure 3:

- Surveillance data acquisition (recording or import) module implemented by IRIS (IOSS and RaSta integrated with SCDB, where IOSS stands for input/output subsystem and RaSta is radar statistics), capable of recording target reports, tracks, and external reference trajectories from other systems in real time into SCDB and extract some elementary statistical figures from raw data (e.g., radar revolution period).

- The new opportunity traffic trajectory reconstruction module performs association, sensor bias (systematic error) estimation and correction [19], and trajectory interpolation. This kind of offline action operates on previously recorded target reports and tracks from SCDB and also saves its results (reconstructed reference tra-

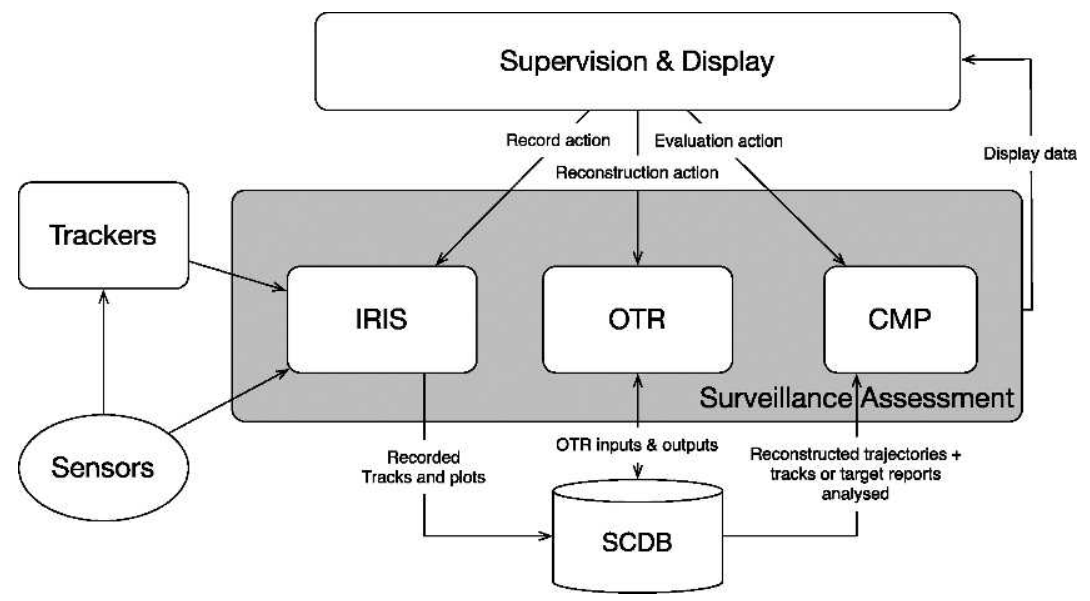

Figure 3.

SASS-C surveillance assessment architecture. 
jectories [RRTs] and others, to be described later) in SCDB.

- Assessments over sensor quality and tracking statistics are performed by surveillance data analysis module (CMP, which stands for CoMParator) module. It uses RRTs from real traffic (provided by OTR) and analyses sensor and tracking behavior (probabilities of detection, false alarms, accuracy, etc.), with a set of statistics adapted to each type of sensor. It also operates on SCDB records in an offline process controlled by supervision.

An example displaying SASS-C input tracks and output RRTs for a given airspace deployment with several radars, can be seen in Figure 4.

\section{ATC RECONSTRUCTION PROBLEM DESCRIPTION}

The core of the ATC system evaluation method implemented by SASS-C is the trajectory reconstruction algorithm (OTR in Figure 3). OTR is able to process measures (target reports) from the following sensors: secondary radars (both conventional and Mode S, using enhanced surveillance ADD [20]), 2D and 3D primary radars;,WAM systems, and ADS-B. Future extensions will cope with automatic dependent surveillance-contract (ADS-C) and airport surface sensors.

To process measurements, time and space alignment of different sensors is necessary, so OTR corrects many types

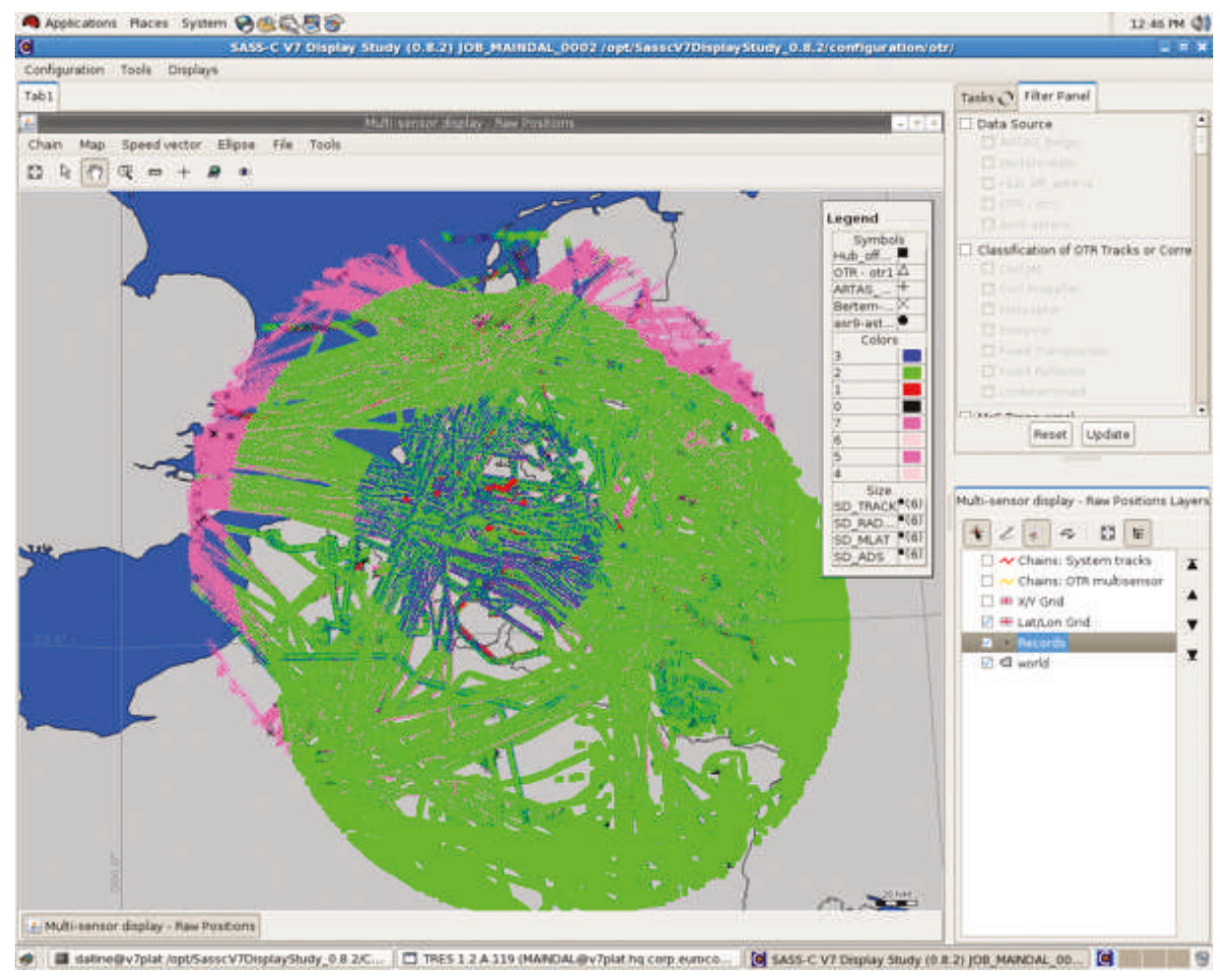

Figure 4.

SASS-C display. of systematic errors, depending on the sensor type. Any remaining bias in the measurements leads to false maneuver detection problems, reducing the quality of the reconstruction results, so complete bias models are derived, and highquality estimation algorithms are implemented.

OTR associates target reports to build reconstructed trajectories, with methods taking into account the measurements accuracy and potential integrity problems. Depending on the sensor, the available data is different (position, speed, identifiers as Mode A or Mode S, height, etc.), and therefore the association procedures must be adapted to the specific features of each data source. After association and bias estimation and correction, smoothing processes are used to obtain a low-error reconstructed trajectory to be used as a reference for the evaluation.

The amount of reports to be processed may be huge, on the order of millions or tens of millions per hour, and some of the processes related to each individual target report can be complex. It is an offline system, and therefore, association, bias estimation, and smoothing need not be performed because target reports are received in an ordered manner in real time. However, there are performance requirements; in general 1 hour of a specified load scenario must be processed in less than an hour in a dual-core Linux ${ }^{\circledR}$ workstation.

OTR also exploits the available geographical information, in the form of sensor coverage, screening files (coverage in elevation), aeronautical information databases, and others, allowing for data filtering at several stages, using either typical database methods or built-in filters (geographical, by sensor, by any code, by type of traffic, etc.).

Finally, main OTR outputs are

-RRTs, defining horizontal position and velocity samples, barometric and geometric height and vertical speeds, MoF segments, and identification codes (Mode $\mathrm{S}$ and Mode A) along trajectory, and classification of RRTs in one of the following types: civil jet, civil propeller, military, helicopter, extreme, fixed field transponder, and fixed reflector.

-associations between input target reports and RRTs.

-correlations between input tracking systems tracks and RRTs.

-sensor noise covariances and bias estimations and intermediate values. They are calculated to enhance reconstruction robustness, but they are also interesting to assess the sensor state. 


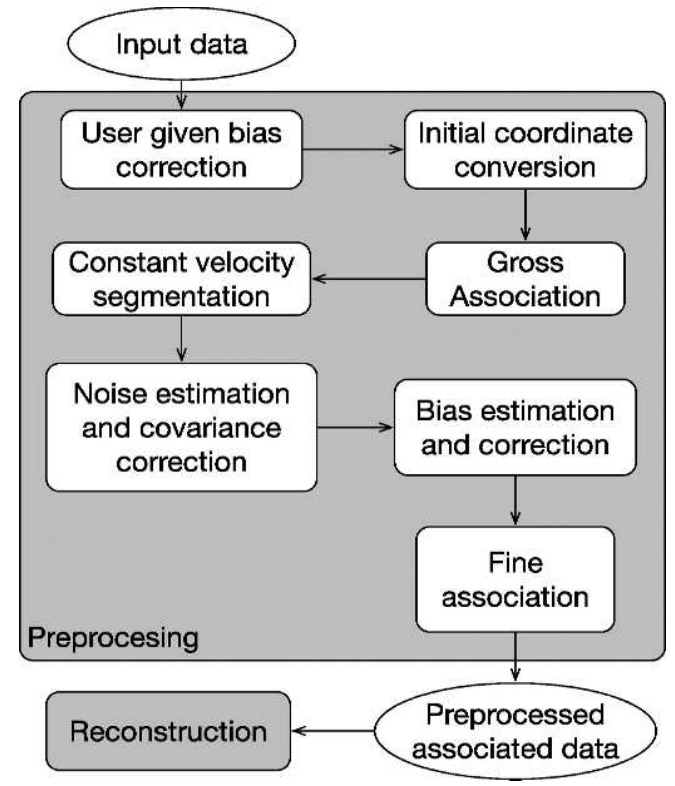

Figure 5.

Preprocessing phases of OTR.

To enhance tool quality, several design decisions were taken. Experience with previous SASS-C versions and with similar real-time tracking systems showed that system site adaptation and installation is a very difficult, costly, and error-prone procedure, as data regarding sensor location and accuracy must be provided. In general, the approach used was defining the minimum amount of parameters to be known and tuned by users, and only defining parameters with a clear physical meaning. Additionally, to enable future extension to other types of sensors, monosensor-multisensor association procedures, bias estimation methods, and reconstruction methods have been highly abstracted.

\section{FUNCTIONAL AND LOGICAL DECOMPOSITION OF OTR}

OTR works as a special "off-line" multisensor fusion system, aiming at estimating target kinematic states based on knowledge of both past and future target reports. There are two main processes involved:

- The preprocessing phase obtains bias-free lists of target reports associated to each trajectory. These target reports must be time and spatially aligned, and the measures from all sensors converted to a common fusion coordinate frame. This process deals with most sensor specific information (Figure 5).

- The 3D reconstruction of the target trajectory is based on previously associated lists of measures.

This process is almost independent of the specific sensors originating the data, with the only exception being some postprocessing enrichments in reconstruction such as correlation with system tracks, analysis of missed reports, and track classification.

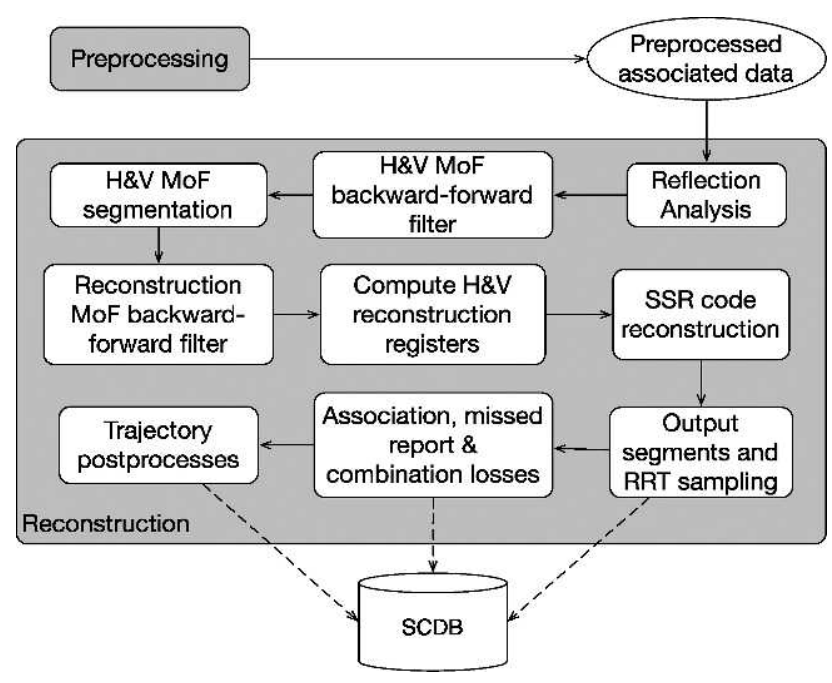

Figure 6.

General architecture for MoF identification, RRT reconstruction, and postprocessing.

The first task consists of transforming all measurements to the same coordinates system and corrects systematic errors of each sensor. To do so, OTR applies user-given biases to correct the measurements and then transform them using stereographic projection [21]. Information about sensor measurement noise is also converted to the noise covariance matrix in central coordinates. Next, OTR may select the data for trajectory reconstruction applying filters specified by SASS$C$ user, which may define $4 \mathrm{D}$ domains of interest (4DoI) and desired plot codes.

The following step has to group sensor data in monosensor tracks related with a target and monosensor tracks among them to form multisensor tracks. This data association process is called gross association and defines a very conservative approach to association, which will be refined in a later process, after bias estimation and correction.

Next, the radar and WAM noise parameters defined by the user must be validated and corrected if the estimated values are not compatible with them. Covariance correction for one sensor demands a recalculation of covariance for all target reports from this sensor. To calculate those noise variances, constant velocity segments measurements are used, and an estimator of the variance is calculated using an observable based on the differences of position in three consecutive measures. So, before noise and bias estimation, OTR searches for segments of rectilinear constant velocity $\mathrm{MoF}$ for each track.

The next process is bias calculation and correction. This is a quite complex system, using data from constant velocity segments of the associated multisensor tracks and mixing the available information to obtain both sensor-related biases (equal for all targets) and target-related biases (dependent on the on-board individual equipment). There is an algorithm exploiting each of these error models, and all of them are related to a common highly abstracted bias estimation architecture. 
After bias correction, data association may be enhanced during a process called fine association, recovering from many ambiguous situations not addressed by gross association.

An additional process performed at several stages of preprocessing is height completion, providing height information for those target reports lacking it. After each association change in gross association or in several stages of fine association, height completion is performed using the most accurate height information available.

After preprocessing, the reconstruction (i.e., smoothing) and postprocessing phases are executed, as depicted in Figure 6.

All internal calculations in the tracking filters are done using the stereographic plane and vertical coordinates, while the final outputs are transformed to geodetic coordinates (latitude, longitude, height) for position and body frame reference for velocity. So the output is $3 \mathrm{D}$, but the problem is divided into two decoupled smoothing processes for horizontal (XY) and vertical (Z) coordinates, as usual in the ATC domain. It should be noted the definition and reconstruction of height or elevation is quite complex, as we have both barometric and geometric height sensors, using mean sea level (MSL) or above ground level (AGL) references.

Before actual reconstruction, a reflection analysis process checks if every multitrack is, in fact, a set of reflected reports instead of a real trajectory, to avoid performing its reconstruction.

The reconstruction process in OTR is based on segmenting the time-ordered sequence of sensor data into homogeneous blocks corresponding to the aircraft motion types (i.e., $\mathrm{MoF}$ ). This information is used later on in order to reconstruct the trajectory generating the segments and samples using specially tuned reconstruction filters for each MoF. Segmentation is not only important for the reconstruction phase but also because many of the accuracy-related statistics obtained by CMP can be filtered taking into account the MoF. Therefore, users can compare the performance of several real-time trackers for a specific MoF. Segmentation and filtering processes are performed in horizontal and vertical dimensions (H\&V in Figure 6).

The last reconstruction process is SSR code reconstruction, which removes erroneous and completes missing values.

The output of the process is MoF segments and RRT samples, which are saved to the database. At the same time, some additional processing to simplify SASS-C CMP module are performed: the presence of loss of detection (missed reports) and primary/secondary combination received from collocated radars with primary and secondary detectors are analyzed. This preprocessed data will be analyzed statistically, after OTR execution, by the SASS-C CMP module to perform a detection analysis process that assesses detection quality of ATC sensors (i.e., probability of detection, false alarm rate, etc.).

Finally, there are several trajectory postprocesses. First, the trajectory is classified. In addition, a gap analysis is used to identify and reconnect trajectories that were separated by long detection gaps. It also includes correlation with system tracks and with external reference tracks (ERTs) provided by other reconstruction systems for comparison purposes.

\section{IMPLEMENTATION OF OTR: KEY DATA STRUCTURES AND PROCESSES}

The key abstracted data within OTR are internal reports, sensor models, association tracks (mono- and multisensor), the list of reconstruction registers, and the RRT, where the data sets are referred to in capital letters and described next.

Internal reports comprise an abstract container for all target reports measures, following a representation similar to that in All Purpose Structured EUROCONTROL Surveillance Information Exchange (ASTERIX) format [22]. It contains basic identification data (key in database, time of measurement, and sensor identification), the raw measurement as provided by the sensor, transformed horizontal measure (in 2D stereographic coordinates), and height used for the coordinate transformation. In addition, it may have height-related information (both for barometric and geometric height) and speed (groundspeed and heading) data if the sensor can provide it. All those kinetic measurements have a random error modeled by means of their associated covariance. This structure also contains the association state of the target report (a flag indicating whether the report was associated or not), the outlier marking flag, and some other internal processing-related flags to preclude its use if there are indications of potential integrity problems. Then, each of the sensor types has a specific derived structure to include additional data (as identification codes for secondary surveillance radar, or Mode S, or amplitude information for primary radars).

Sensor models are defined through a sensor type, a reference position (i.e., radar position) necessary for coordinate transformations, integrity related tests, and measurement period (for periodic sensors). It contains abstracted methods to perform coordinate transformation, horizontal and vertical covariance calculation (for position and velocity, if available), and to aid in the estimation and correction of noise covariances and biases (which are sensor-type specific). Again, there are subclasses for each type of sensor model, implementing the abstracted interfaces.

Association tracks are of two types: monosensor and multisensor tracks. They are composed of a list of time-ordered internal reports, a list of constant velocity segments (derived in preprocessing and used for noise and bias estimation), and identification data (Mode A, in consecutive time intervals, and a unique Mode $\mathrm{S}$ address). There are specific sensor-type structures for each type of monosensor tracks. Those association-related tracks contain abstract methods usable as part of the highly abstracted gross and fine association procedures. Multisensor tracks also contain a unique key for reference in the database and target-related biases for some sensor types (ADS-B, secondary, and Mode S radars). 


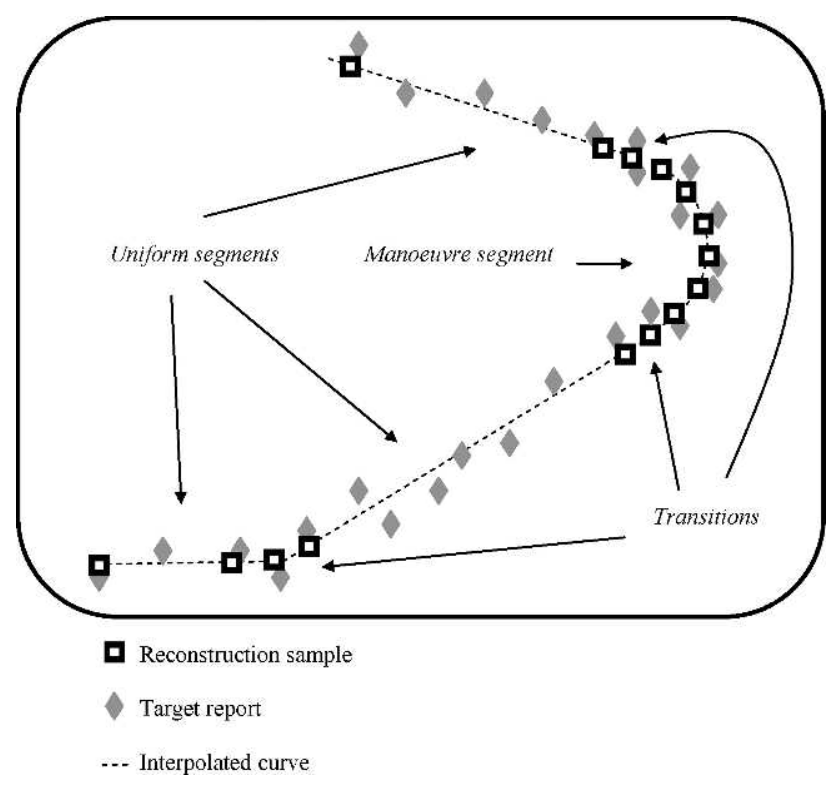

Figure 7.

Representation of a reconstructed curve with discrete samples.

There is another intermediate data structure, used for the MoF segmentation, 3D reconstruction, and postprocessing phases: the list of reconstruction registers. It is a time-ordered list of registers containing all the reconstruction information to synthesize the reference output. Each reconstruction register contains the estimated parameters resulting from forward and backward filtering runs (mode probability, position, velocity, vertical velocity, smoothed code, etc.), and they are synchronized in time with associated internal reports within a multisensor association track.

The RRT is defined through horizontal and height MoF segments, identification codes (Mode A, potentially different in successive time intervals, and Mode $S$ address), and reconstruction samples. The sampling frequency is variable, as depicted in Figure 7, depending on the MoF: less samples in uniform segments and more during sharp maneuvers. In the case of nonclassified segments (noise, high maneuvers, etc.), where no dynamic model can be exploited, the samples are in direct correspondence with target reports. The objective is simplifying the interpolation means used by later processes within SASS-C, such as CMP, which uses a simple linear interpolation exploiting position and velocity from samples. Those samples not only include 3D kinematic information but also reconstruction quality in the form of associated covariance matrices.

\section{BIAS ESTIMATION}

The key point in the design of a bias estimation and correction subsystem is the identification and modeling of the dominant error terms in the measurement process. Bias terms can be divided in two subtypes: sensor-related bias, which have the same value regardless of the target; and target-related bias equal for every sensor of the same type. OTR bias estimation is based on generic bias estimation architecture for multisensor-multitarget surveillance systems. It first performs bias estimations from measurements from each target of a subset of sensors assumed to be reliable and then combines all the bias estimation information from all the targets to obtain, for each sensor, its corresponding global bias. Afterwards, sensor global bias terms are corrected to subsequently calculate the target specific biases. Once the target biases are corrected, the process is repeated for other sets of less reliable sensors, assuming bias-corrected measures as unbiased. The bias components defined for OTR-related sensors were

- for radars: range bias, range gain, azimuth bias, azimuth eccentricity, and time offset between sensors. Additionally, for secondary radars, response delay offset, which is a target-related bias, is estimated. Those are classical bias terms for radar [20], [23], [24]. In future versions, extensions, such as barometric to geometric height offset estimation and correction, will be included.

- for ADS-B: time and position offset, different for each aircraft (target biases). Most ADS-B targets provide global positioning system (GPS) or differential GPS (DGPS) measurements, which typically suffer from a potential time jitter and offset, limited by ADS-B standard [25]. Time-varying GPS errors are smaller than ADS-B quantification errors; in general, the convergence of the estimators for related target bias estimators would be too slow to perform effective correction of those terms.

- for WAM: a map/grid with position offsets depending on the position, and a time bias, common for all targets (sensor bias). It is not feasible to use a more complex model related to actual measurement error sources, such as propagation or station clock drift [26], due to the lack of knowledge of the positions of the stations providing the measurement, potentially changing from measure to measure due to interference (garbling) and coverage effects. The information regarding the used stations cannot be recovered from currently defined ASTERIX reports, so a generic interpolation bias model is used.

In OTR initial design phases (by year 2006), radar data were assumed to be more reliable, due to the longer operational experience and to the availability of more consistent bias models.

- First, radar data are used to estimate radar biases (first sensor biases, then target biases).

- Then, corrected radar data and uncorrected ADS-B data are used to estimate ADS-B biases.

- Finally, corrected radar data and uncorrected WAM data are used to estimate WAM biases.

The resulting processing order is the one shown in Figure 8. 
Additional details on the bias calculation algorithms can be found in [27] and [19]. They were designed in a modular way so that a change in the processing order can be performed for future versions of OTR.

\section{ASSOCIATION}

Association in OTR is divided in two processes: gross association and fine association. Gross association is performed in advance of bias estimation and correction, and therefore, it is very conservative, only allowing associating measures in areas with low false alarm rates and without aircraft crossing. The objective of this phase is obtaining multisensor association tracks enabling noise and bias estimation. Typical real data scenarios have only $80-90 \%$ of target reports associated after this phase.

On the other hand, fine association is able to associate 98-99.7\% of target reports in typical scenarios with correctly behaving sensors (which include the presence of false alarms, splits, etc.). Its objective is associating all available data to obtain RRTs covering the full trajectory in the sensor network coverage. It is especially important to have all target reports related to the target entering and leaving the sensor coverage correctly associated and to be able to assess sensor coverage size and shape and real-time tracker initialization delays.

Gross association is performed in two parallel processes, which are performed over consecutive blocks of time-ordered data:

- Monosensor track association. It is different depending on the sensor type, running in parallel for all sensors. The process has been abstracted so that the differences for each sensor only concern certain constants as gate sizes, the number of measures to confirm a track, and the need to accomplish identification compatibility checks. It builds 2D horizontal Kalman filter tracks capable of tracking maneuvering targets and calculates samples at predefined time instants. Those samples also contain height information, if available in the measures.

Multisensor track association. It uses the monosensor tracks, sampled at common time instants, to merge them into a multisensor track, if the kinematic estimates from the Kalman filters are statistically compatible, taking into account the potential presence of measurement biases. Additionally, it performs tests on height and code (Mode A and Mode S) compatibility.

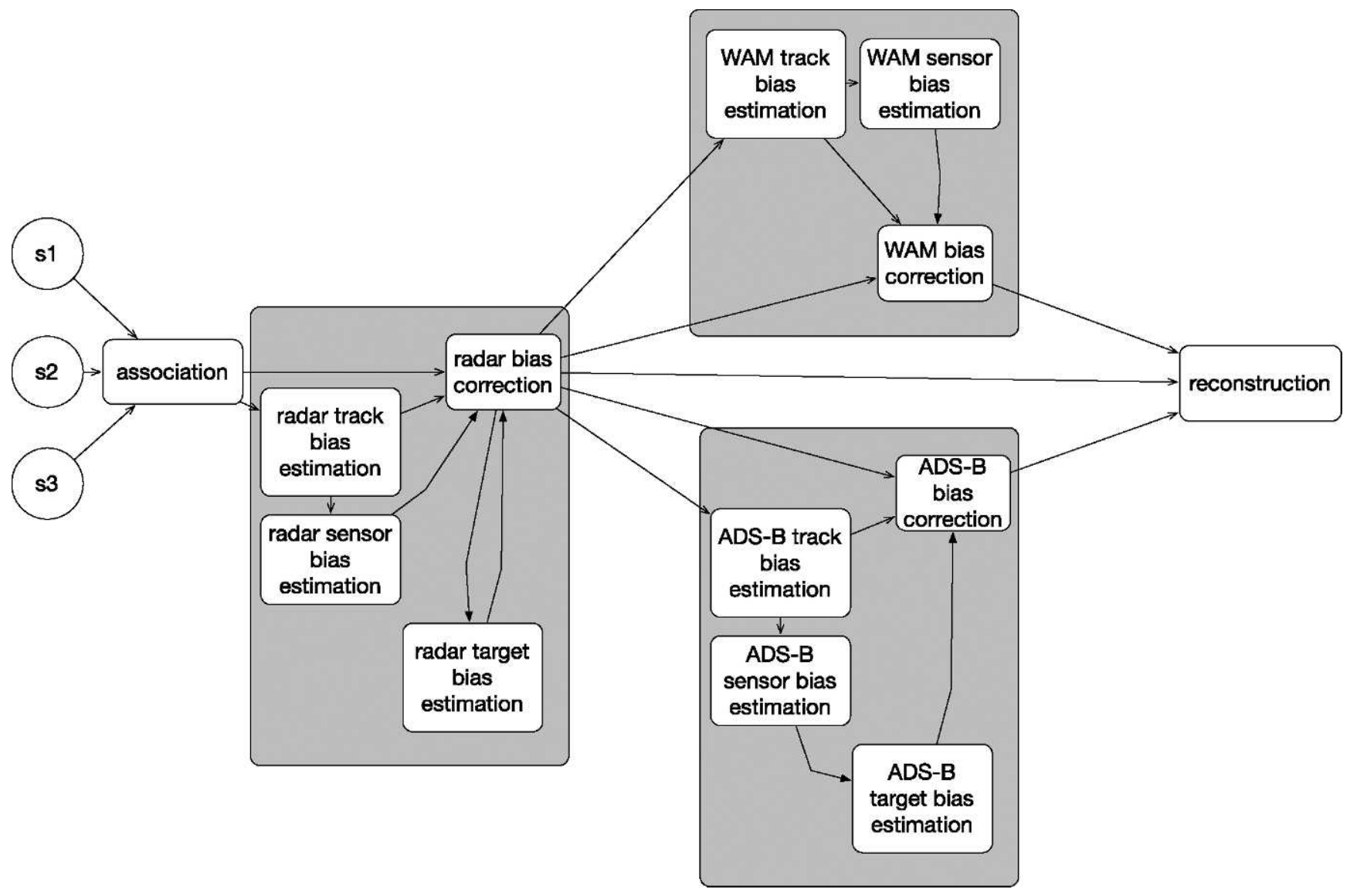

Figure 8.

Bias estimation and correction process. 
There are limits for minimum track length (number of measures, lifetime length) of the monosensor tracks to be used for multisensor track association, ensuring only long (stable) tracks will be created in these first stages of the association algorithm. It takes into account code information (Mode A, Mode S), position compatibility, time of measurement compatibility, and velocity compatibility among monosensor tracks.

This process is similar to a real-time tracking system process but with less requirements over the output. So, each time there is some kind of ambiguity in association (two target reports potentially feeding the same track or two competing monosensor tracks are too near), it splits the tracks in smaller unambiguous segments. Multisensor association gates are quite big, in order to allow association in worst-case bias, and track stability is demanded to avoid erroneous association potentially corrupting bias estimation processes. It should be noted that the gross association not only provides nonproblematic multisensor tracks, but it also provides shorter monosensor tracks (to be called small tracks), with even only one measure, to be used for fine association procedures. Those small tracks contain all target reports not associated with multisensor tracks along gross association.

After bias estimation, OTR performs a new association procedure (fine association). It is based on target report time-space compatibility, with reduced association gates, as bias was previously corrected. It is performed by means of a plot assignation voting procedure using gross association tracks as initial seeds and finding compatible small tracks in the vicinity. Also, previous tracks may be merged into one, solving potential identification or bias-induced gaps not resolved during gross association. Some small tracks, under certain situations, may also become seeds for the fine association process, escalating to multisensor track status. Depending on sensor quality, percentages of nonassociated target reports on the order of $0.3-2 \%$ are attained after this process, with quite robust behavior even during trajectory crossings or aircraft in areas with false alarms. Primary radar with clutter remains the most problematic sensor, due to its lack of target report identification capability.

\section{HEIGHT COMPLETION}

There are several different height sources, depending on the available data. The OTR approach includes the definition of three different kinds of heights:

- Barometric height: can be obtained from Mode C information and may also be provided by ADS-B.

- Geometric height: can be obtained from different data sources, such as 3D primary radar, WAM, or certain implementations of ADS-B.

- Transformation height: to be used for the transformation of radar raw measures to the stereographic plane.
The general approach for height processing is based on the independent reconstruction of barometric and geometric heights (if available) and on the definition of the transformation height from several data sources, using the first available data in the following list:

- Geometric height in the measure.

- Barometric height in the measure.

- Geometric height in surrounding measures associated to the same track.

- Barometric height in the surrounding measures associated to the same track.

- A fixed reconstruction sensor-dependent height.

\section{TRAJECTORY SEGMENTATION}

A key point in the reconstruction phase is the determination of the MoF segments composing a trajectory.

In OTR MoF, segments (including associated qualities) are computed using interacting multiple mode (IMM) filtering [28] for the horizontal plane and adaptive Kalman filtering for the vertical coordinate over all target reports associated with the multisensor track, operating in time-increasing (forward) and time-reverse (backward) directions. These filters derive the horizontal mode probabilities and vertical vectors containing the estimated height and vertical velocity and accelerations in the reconstruction registers. Changes in probability modes or in vertical speed are associated to changes in $\mathrm{MoF}$ and allow the segmentation of the flight. Finally, horizontal segments are enhanced fusing segments derived from an additional source: measured velocities, contained in aircraft-derived data (ADD) registers, if available in the target reports. This kinematic data is processed to find nonzero accelerations using a Kalman filter and an adaptive threshold, detecting maneuvering segments. In the same way, vertical MoF segments are obtained by the fusion of segments corresponding to barometric and geometric height, if sources of both types of data are available. Also, a test over tracker residuals is used to detect the presence of outliers and so called high-energy maneuvers (maneuvers with very high and rapidly changing accelerations that cannot be accurately reconstructed with the nominal smoothing techniques).

Once the segments are calculated, trajectory matching is performed for some segments in order to label them as one of the predefined MoFs and obtain related kinematic parameters. This matching is based on a statistical pattern recognition process in which the data is interpolated using several candidate MoF canonical trajectories (constant acceleration turn and constant longitudinal acceleration or deceleration), and the accumulated difference between the measures and the interpolated curve is compared to an adaptive threshold taking into account measurement quality.

As a result of segmentation, the trajectory is divided into MoF sections, each one containing the associated time in- 


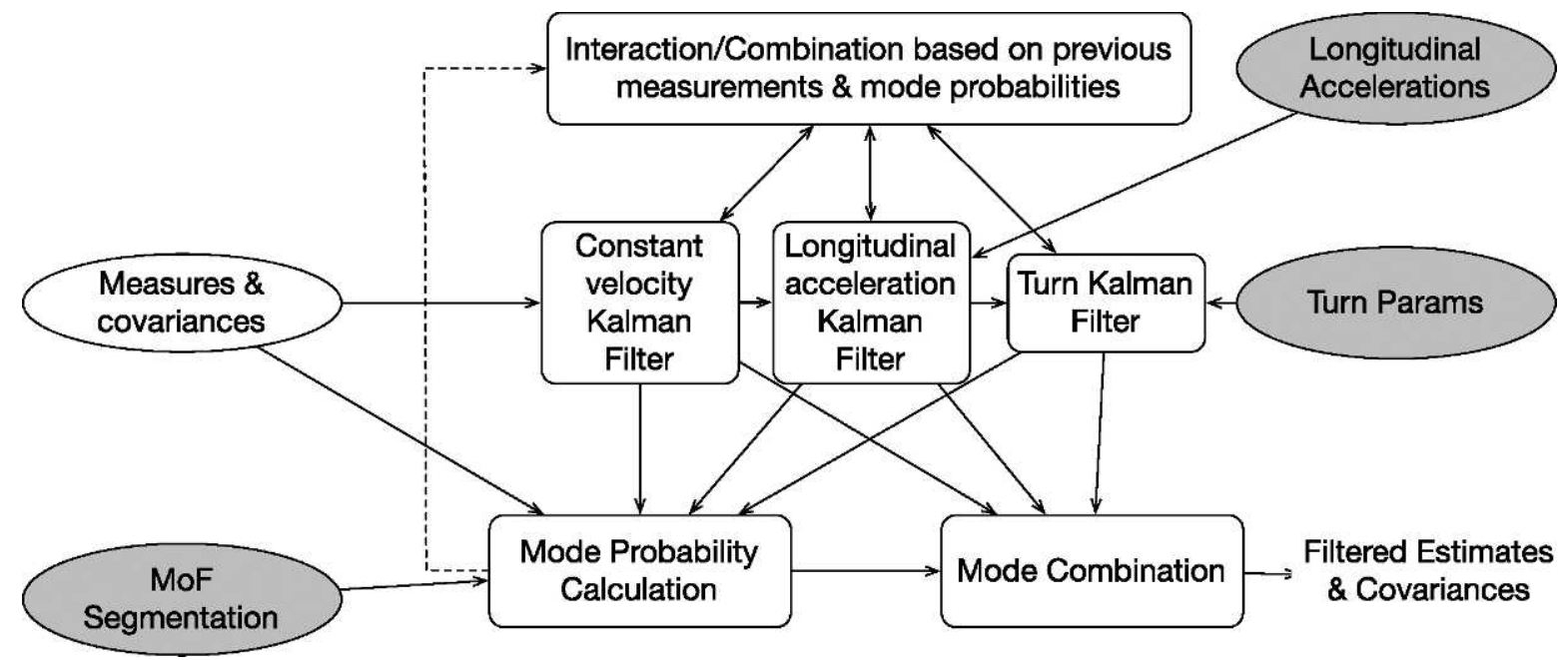

Figure 9.

IMM structure for RRT reconstruction.

terval, segment type, and additional kinematic parameters. The segment types in horizontal plane are "unknown," "uniform," "constant acceleration transversal maneuver," and "constant acceleration longitudinal maneuver." In the vertical coordinate, the segment types are "unknown," "constant altitude," and "constant vertical velocity."

\section{TRAJECTORY RECONSTRUCTION}

Trajectory reconstruction carries out a smoothing process over available target reports, based on mixing the estimates of modified forward and backward IMM tracking filters, which take into account the estimated kinematic parameters of each MoF segment (see Figure 9, where the grey elements denote the new inputs and their effect in a classical IMM schema). This smoothing process is designed to guarantee continuity of reconstruction with an appropriate trade-off between noise reduction and adaptation to maneuvers. This new double pass computes the state vectors (position and velocities), where various segments types have different dynamic modes in the IMM filters, with the corresponding parameters (accelerations, turning radius, etc.). In the vertical dimension, specially tuned reconstruction forward and backward Kalman filters, for each type of segment, are also defined. In addition, vertical smoothing is corrected through a nonlinear process to avoid the overshooting effects in maneuvers, by giving more weight to the appropriate filter (forward or backward) at each side of the maneuver.

Reconstruction registers are obtained combining the reconstruction horizontal IMM forward and backward states in the horizontal plane and combining the vertical Kalman forward and backward states (using a parallel procedure to horizontal reconstruction).

Reconstruction filters take advantage of the ADD if available. In this case, the velocity measurements (groundspeed and heading) are projected onto the stereographic plane and integrated in each Kalman filter of the reconstruction IMM.
To avoid instabilities due to corrupted velocity information, several consistency checks are performed over these ADD before including them in the smoothing process.

The reconstruction along not successfully classified segments is done with a generic and robust mode with default parameters, keeping the same IMM architecture. Besides, the filtering method is protected against outliers and highenergy maneuvers in the estimation of position and velocity. More details on this process may be found in [29].

\section{POSTPROCESSING}

After obtaining the reconstructed trajectories, some additional processes to prepare the data for its exploitation by CMP are performed. Next, we will detail them:

- Classification of aircraft category: it is based on the application of a set of rules to the history of reconstructed trajectory velocity and accelerations. This can be exploited by CMP to perform aircraft class-oriented filters, to obtain statistics of relevance for each particular application, and to remove not relevant types of targets from the analysis. The techniques used here are similar to proposals presented in [30] to analyze the trajectory as sequences of homogeneous segments or in [31] and [32], which extract tracking features for classifying the motion patterns of interesting entities. According to the type and magnitude of maneuvers, together with availability of other features such as the secondary code, the trajectory is classified accordingly in the following categories: \{civil jet, civil propeller, military, helicopter, extreme, fixed field transponder, fixed reflector\}.

- Large-gap analysis and reconnection: the reconnection process detects the presence of gaps in the reconstructed trajectories, usually due to coverage gaps. This process is done with a global analysis searching for sets 


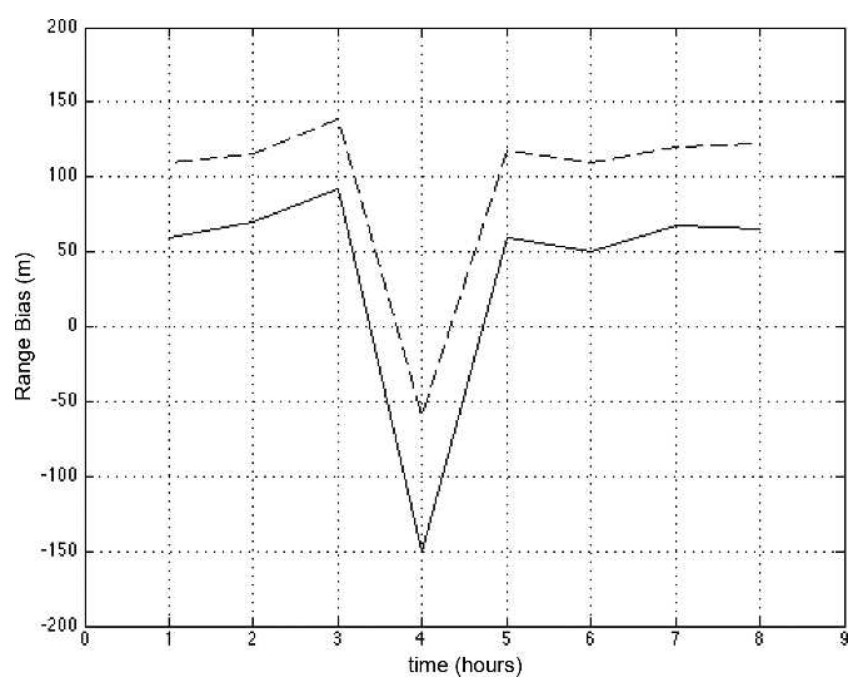

Figure 10.

Radar range bias estimation results.

of RRTs that could be reconnected, using compatibility tests over maximum horizontal and vertical separation, potential changes in speed and heading, and compatible identification codes (Mode A and especially Mode S). This subsystem was designed to avoid the reconnection of sections with slight variations in speed and heading, but at the same time avoiding the undesired effect of connecting trajectories from different targets.

- System track correlation: many CMP analyses of tracker performance are based on the comparison of the RRT with a track provided by the real-time tracker. So, an extremely important feature of OTR is its capability to provide RRTs correlated with those tracks. It should be noted that this is in general not a simple problem, as there may be several tracks related to an RRT, especially if there are gaps or problems in association due to crossing targets or very near parallel targets. A certain RRT can be, therefore, correlated with several tracks, and the opposite is also possible, the same track may be correlated with several RRTs. The OTR solution is based on defining time segments for the correlation so that the correlation is one-to-one within any given time segment. Correlation is based on distance assessments of kinematic magnitudes (position and velocity), taking into account their associated quality, expressed in terms of covariance.

- External track enrichment: RRTs resulting from the reconstruction process can be used as a source to enrich external reference tracks (ERTs), provided by outer high-quality telemetry systems. This process requires two tasks: ERT correlation (similar to system track correlation) and ERT enrichment. After correlation, an ERT may be enriched with the RRT samples and additional information to produce a new structure, which is stored as a new RRT output in the SCDB.

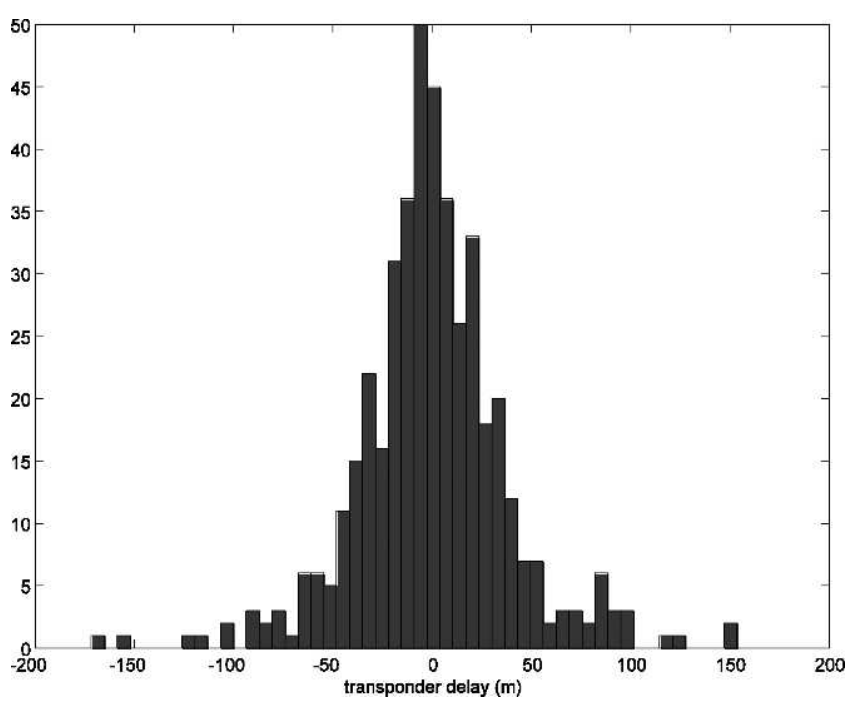

Figure 11.

Transponder delay estimates histograms for real secondary radar traffic.

\section{RESULTS}

Figure 10 shows the result of two radars' bias estimation from real data sets from a scenario containing more than 10 Mode S, secondary, and primary radars, divided into 1-hour exercises. The bias was estimated during eight successive hours, in order to show bias estimation stability. The figure shows range bias in meters of two secondary radars.

In these data there was a clear change in the estimation for hour number four. After investigating the results, it became apparent that there had been a recording problem with the time stamping of several radars data (there was an offset exceeding 10 seconds for a certain subset of the radars and for a certain time interval comprising measures for this hour); therefore, the constant bias assumption did not hold. These results indicate that the reconstruction will suffer if this kind of erratic nonstationary behavior appears in the measures, but bias estimates for the rest of the hours are quite similar, for all bias terms, demonstrating the stability and consistency of the algorithms.

Another indication of the consistency of the estimation is the distribution of the target bias. For instance, if the radar bias is not estimated in a consistent way, transponder delay estimates will become larger, and their distribution skewed. Figure 11 depicts a histogram of transponder delay estimates for a real scenario with nearly a thousand aircraft.

From the obtained results, the delay estimates show a distribution compatible with a transponder 75-m maximum delay error requirement from ICAO standards [18]. The estimates have approximately a zero mean, and there are some aircraft with a larger transponder delay (to be investigated).

Next, some illustrative examples of the reconstruction of real flights will be included. In general, the reconstruction shows effective smoothing of the trajectory for constant velocity segments, fast and accurate detection of changes in $\mathrm{MoF}$ (maneuvers), and the lack of overshooting effects [29]. 


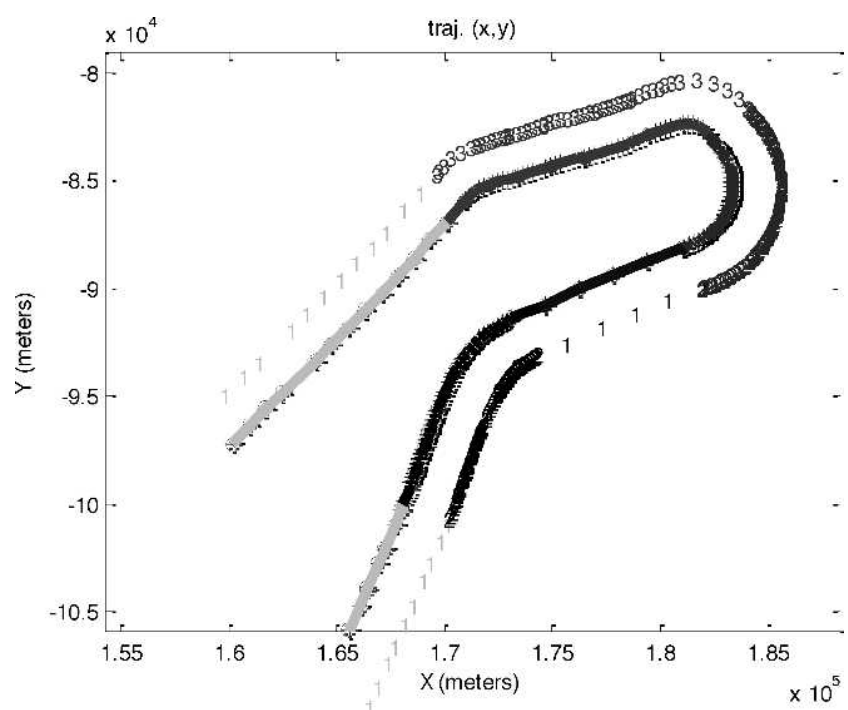

Figure 12.

Horizontal MoF segmentation, trajectory 1.

In Figures 12 and 13, the results of MoF segmentation in two real trajectories can be appreciated.

The numbers associated represent the horizontal MoF (1 stands for uniform motion, 2 for turns, and 3 for longitudinal acceleration). The trajectories contain uniform sections and intervals with changes in heading or groundspeed (similar to the typical maneuvers in approach and landing operations), which are detected with a very acceptable result.

With respect to vertical reconstruction, Figure 14 illustrates a real trajectory reconstruction, containing the smoothed curve and vertical MoF (1 stands for constant height and 2 for climb/descend maneuver).

In the detail of descent-uniform transition in Figure 15, we can appreciate the smoothed result. Note the discretization effect in the barometric flight levels in the input to vertical reconstruction and the lack of overshoot in the transitions to vertical maneuvers in the smoothed result.

Regarding gap analysis and reconnection, the following example (Figure 16) shows the identification and reconnection of sections from the same trajectory. It describes racetracks in a zone with partial radar coverage, indicating detection gaps of around 60 seconds per turn.

\section{CONCLUSIONS AND FUTURE RESEARCH}

The presented system supposed a complete redesign of the SASS-C reconstruction suite, especially of the trajectory reconstruction process. The main innovative features of this system with respect to the previous generations of SASS-C reconstruction systems were

- splitting of association problem in two phases (i.e., gross association, before bias estimation; and fine association, after bias estimation and correction) possible due to the offline processing nature of SASS-C.

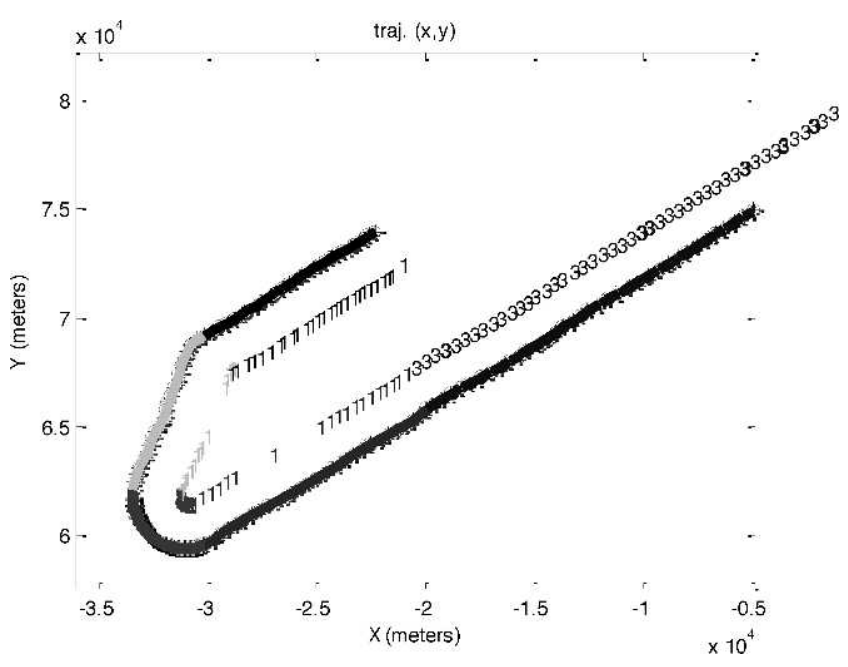

Figure 13.

Horizontal MoF segmentation, trajectory 2.

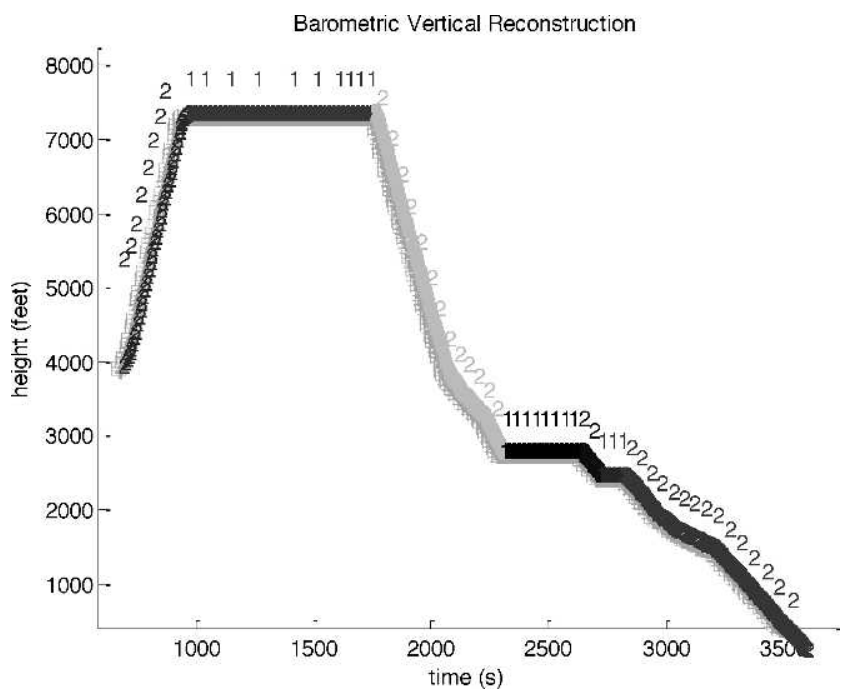

Figure 14.

Vertical reconstruction.

- complete and comprehensive bias estimation procedure for all sensors.

- exploitation of aircraft dynamics in reconstruction algorithms, based on a two-stage process: MoF segmentation and adaptive IMM/Kalman smoothing.

Its highly abstracted functionalities may form the basis for future improvements in bias estimation, inclusion of data processing methods for new sensors, and new reconstruction algorithms. The paper tries not only to describe the reconstruction system but also to clarify the key aspects to be taken into account while building reference trajectories (which can be translated to many other fields of application) and emphasizes the differences with real-time trackers. Those differences derive from noting that in this kind of batch processing all past and future measurements are available. Therefore, major changes in all data processing func- 


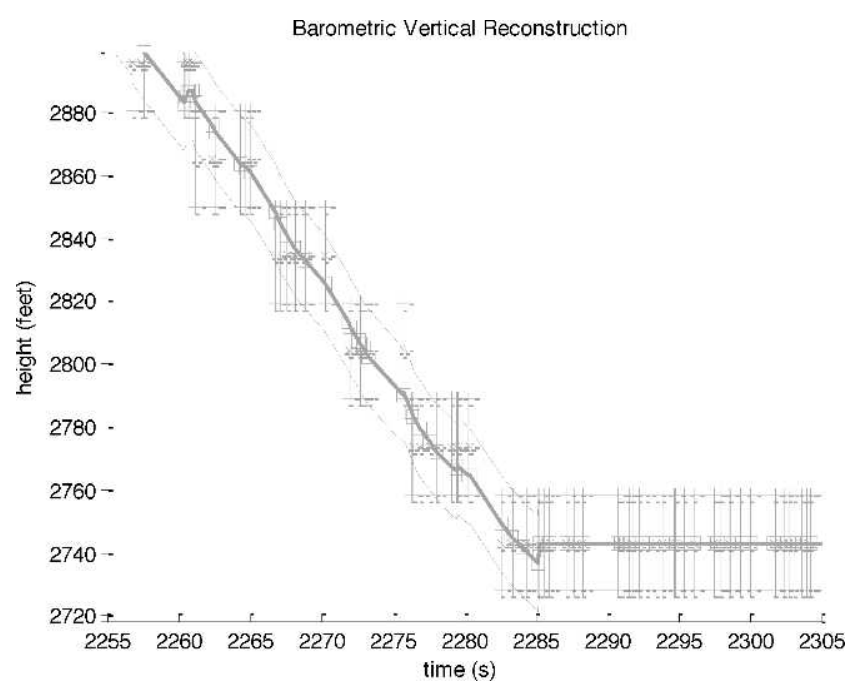

Figure 15.

Vertical reconstruction (detail).

tions, and even in the overall system architecture, should be done to actually attain the increased trajectory estimation quality needed to be able to assess online tracker quality.

Future research and system improvements, related to SASS-C, will cover several aspects:

- Adaptive OTR: we are working on automatically recognizing problematic sensors and adapting reconstruction to this identification, by removing their problematic data from the data fusion process, or modifying the processing order, for instance, in bias estimation. The idea is improving overall OTR result integrity.

- Improvements to bias estimation once operational expertise is gained with new sensors. The processing order in Figure 8 could be changed, especially if improved bias models are integrated for WAM and ADSB.

- Incorporation of new sensors from airport surveillance and adaptation of reconstruction algorithms for this area. This aspect is important if we tend to see the trajectory as defined from gate-to-gate, in line with current trajectory-based operation air traffic management paradigms, as the ones being researched within Single European Sky ATM Research (SESAR) or Next Generation Air Transportation System (NextGen) programs.

The adaptation of these techniques to other applications, with appropriate problem-specific solutions, seems a feasible and cost-effective solution to the always challenging problem of multisensor-multitarget tracking quality assessment in nonstationary operational environments.

\section{ACKNOWLEDGMENT}

This work was funded by contract EUROCONTROL's TRES, by the Spanish Ministry of Economy and Competitive-

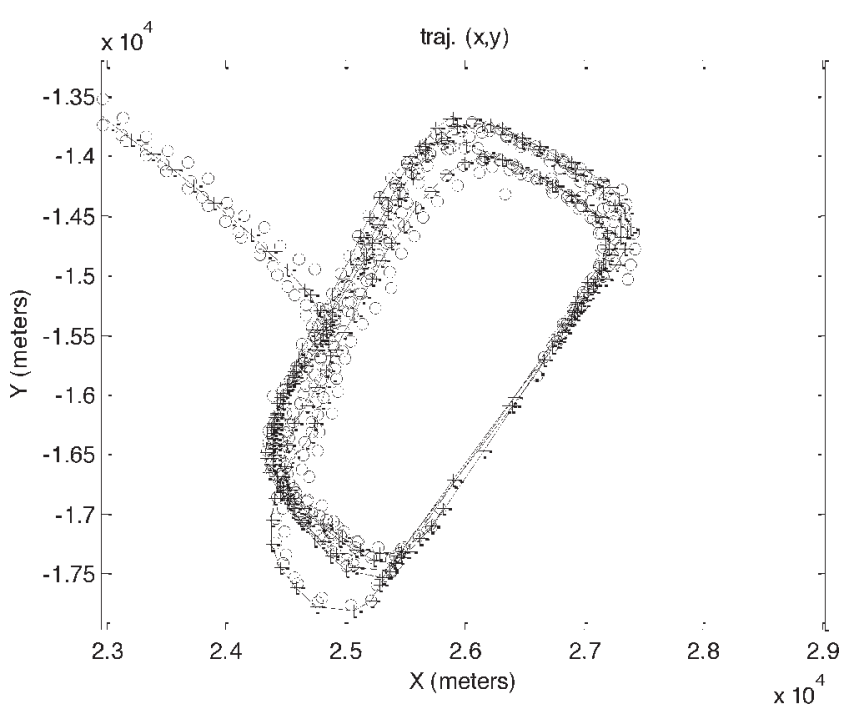

Figure 16.

RRT gap reconnection.

ness under grants CICYT TEC2008-06732/TEC and CYCIT TEC2011-28626, and by the Government of Madrid under grant S2009/TIC-1485 (CONTEXTS).

\section{REFERENCES}

[1] Donohue, G. A visionary look at aviation surveillance systems. IEEE Aerospace and Electronic Systems Magazine, Vol. 10, 10 (1995), 8-14.

[2] Galati, G., Leonardi, M., and Tosti, M. Multilateration (local and wide area) as a distributed sensor system: lower bounds of accuracy. In Proceedings of the 5th European Radar Conference, Amsterdam, the Netherlands, October 30-31, 2008, 196-199.

[3] Stevens, M. C. Secondary Surveillance Radar. Norwood, MA: Artech House, 1988.

[4] O'Hern, B., Mack, J., Pieramico, A., Rugger, D., and Moyer, L. The Radar Support System (RSS): a tool for siting radars and predicting their performance. In Proceedings of the IEEE National Radar Conference, Syracuse, NY, May 13-15, 1997, 211-216.

[5] Menington, I. S., and Green, G. M. Measurement and interpretation of radar system performance in a complex environment. In IEE Colloquium on Specifying and Measuring Performance of Modern Radar Systems (Ref. No. 1998/221), London, United Kingdom, March 6, 1998, 4.

[6] Blasch, E. P., Pribilski, M., Daughtery, B., Roscoe, B., and Gunsett, J. Fusion metrics for dynamic situation analysis. Proceedings of SPIE, Vol. 5429 (2004), 428-438.

[7] Blackman, S., and Popoli, R. Design and Analysis of Modern Tracking Systems. Norwood, MA, Artech House, 1999.

[8] Mori, S., Chang, K. C., and Chong, C. Y. Performance analysis of optimal data association with application to multiple target tracking. In Multitarget-Multisensor Tracking: Applications and Advances, Vol. 2, Y. Bar-Shalom, Ed. Norwood, MA: Artech House, 1992, ch. 7.

[9] Mori, S., Chong, C.-Y., and Chang, K. C. Performance prediction of feature-aided track-to-track association. In Proceedings of the 
14th International Conference on Information Fusion, Chicago, IL, July 5-8, 2011, 1-8.

[10] Li, X. R., and Bar-Shalom, Y. Performance prediction of the interacting multiple model algorithm. IEEE Transactions on Aerospace and Electronic Systems, Vol. 29, 3 (1993), 755-771.

[11] Yang, C., and Blasch, E. Characteristic errors of the IMM algorithm under three maneuver models for an accelerating target. In Proceedings of the 11th International Conference on Information Fusion, Cologne, Germany, June 30-July 3, 2008.

[12] Chang, K. C., Song, Y., and Liggins, M. E. Performance modeling for multisensor data fusion. Proceedings of SPIE, Vol. 5096 (2003), 354.

[13] Chong, C. Y. Problem characterization in tracking/fusion algorithm evaluation. IEEE Aerospace and Electronic Systems Magazine, Vol. 16, 7 (2001), 12-17.

[14] Heidger, R., and Nguyen, H. S. An analysis working position for radar data processing quality control. In Proceedings of the Enhanced Solutions for Aircraft and Vehicle Surveillance 2007 Conference, Bonn, Germany, 2007.

[15] Renes, J. J., Kraan, P. v. d., and Eymann, C. Flightpath reconstruction and systematic radar error estimation from multiradar range-azimuth measurements. In Proceedings of 24th IEEE Conference on Decision and Control, 1985, 1282-1285.

[16] Renes, J. J., and Best, M. R. Theoretical background to the reconstruction facility MURATREC. National Aerospace Laboratory, Amsterdam, the Netherlands, Rep. NLR TR 84008 L, 1984.

[17] Blasch, E., Kadar, I., Hintz, K., Biermann, J., Chong, C., and Das, S. Resource management coordination with level 2/3 fusion issues and challenges. IEEE Aerospace and Electronic Systems Magazine, Vol. 23, 3 (Mar. 2008), 32-46.

[18] Hanselman, P. B., Lawrence, C., Fortunano, E., Tenney, R. R., and Blasch, E. Dynamic tactical targeting. Proceedings of SPIE, Vol. 5441 (2004), 36.

[19] Besada, J. A., de Miguel, G., Bernardos, A. M., and Casar, J. R. Generic multisensor multitarget bias estimation architecture. IET Radar, Sonar and Navigation, Vol. 6, 5 (June 2012), 365-378.

[20] Stevens, M. C. Secondary Surveillance Radar. Norwood, MA: Artech House. 1988.

[21] Torge, W. Geodesy (3rd ed.). Berlin, Germany: Walter de Gruyter, 2001.
[22] All Purpose Structured EUROCONTROL Surveillance Information Exchange (ASTERIX), http://www.erocontrol.int/asterix/public/subsite_homepage/homepage.html.

[23] Barton, D. K. Modern Radar System Analysis. Norwood, MA: Artech House. 1988.

[24] Fischer, W. L., Muehe, C. E., and Cameron, A. G. Registration errors in a netted air surveillance system. MIT Lincoln Laboratory, Lexington, MA, Technical Note 1980-40, 1980.

[25] RTCA. Minimum Operational Performance Standards for 1090 $\mathrm{MHz}$ Extended Squitter Automatic Dependent SurveillanceBroadcast (ADS-B) and Traffic Information Services-Broadcast (TIS-B). RTCA, Washington, DC, RTCA document DO-260A, 2006.

[26] Leeson, M. J. Error analysis for a wide area multilateration system. QinetiQ/C\&IS/ADC/520896/7/19 (2006).

[27] Besada, J., de Miguel, G., Soto, A., and Bernardos, A. Algorithms for opportunity trajectory reconstruction. In Proceedings of the Tyrrhenian Internation Enhanced Surveillance of Aircraft and Vehicles, Capri, Italy, September 3-5, 2008.

[28] Blom, H. A. P., and Bar-Shalom, Y. The interacting multiple model algorithm for systems with Markovian switching coefficients. IEEE Transactions on Automatic Control, Vol. 33, 8 (1988), 780-783.

[29] Garcia, J., Soto, A., de Miguel, G., Besada, J., and Tarrio, P. Trajectory reconstruction techniques for evaluation of ATC systems. In Proceedings of the Tyrrhenian Internation Enhanced Surveillance of Aircraft and Vehicles, Capri, Italy, September 3-5, 2008.

[30] St-Hilaire, M.-O., Lefebvre, E., and Helleur, C. Track modeling for maritime surveillance. In Proceedings of the 11th International Conference on Information Fusion, Cologne, Germany, June 30July 3, 2008.

[31] Rhodes, B. J., Bomberger, N. A., and Zandipour, M. Probabilistic associative learning of vessel motion patterns at multiple spatial scales for maritime situation awareness. In Proceedings of the 10th International Conference on Information Fusion, Quebec, Canada, July 9-12, 2007.

[32] Brax, C., Karlsson, A., Andler, S. F., Johansson, R., and Niklasson, L. Evaluating precise and imprecise State-Based Anomaly detectors for maritime surveillance. In Proceedings of the 13th International Conference on Information Fusion, Edinburgh, Scotland, July 26-29, 2010. 